\title{
Accumulation of electric-field-stabilized geminate polaron pairs in an organic semiconductor to attain high excitation density under low intensity pumping
}

\author{
N. C. Giebink \\ Princeton Institute for the Science and Technology of Materials (PRISM), Princeton University, \\ Princeton, New Jersey 08544 and Department of Electrical Engineering, Princeton University, Princeton, \\ New Jersey 08544 \\ S. R. Forrest ${ }^{\text {a) }}$ \\ Department of Electrical Engineering and Computer Science, University of Michigan, Ann Arbor, Michigan \\ 48109; Department of Physics, University of Michigan, Ann Arbor, Michigan 48109; \\ and Department of Materials Science and Engineering, University of Michigan, Ann Arbor, \\ Michigan 48109
}

(Received 1 September 2006; accepted 26 September 2006; published online 8 November 2006)

The recombination dynamics of geminate polaron pair (PP) states are investigated by monitoring electric-field-induced delayed fluorescence in thin films consisting of the green laser dye, Coumarin-6 (C6) doped at $1 \mathrm{wt} \%$ into 4,4'-bis( $N$-carbazolyl)biphenyl. We find that the PP decay follows $\tau^{-m}$ (with $m \sim 0.1$ ), where $\tau$ is the time that the PPs are held in the field. This sublinear decay suggests the possibility for accumulation of PPs over time that can then be reconverted into excitons upon field removal. We demonstrate the generation of short ( $\sim 50 \mathrm{~ns}$ full width at half maximum) bursts of C6 fluorescence with peak intensities $>20$ times the steady-state fluorescence intensity (corresponding to a C6 singlet exciton density $N_{S}>4 \times 10^{15} \mathrm{~cm}^{3}$ ) when pumped continuously by a low intensity $\left(<1 \mathrm{~W} / \mathrm{cm}^{2}\right)$ laser in the presence of a pulsed electric field. (C) 2006 American Institute of Physics. [DOI: 10.1063/1.2385840]

The past several years have witnessed substantial progress $^{1,2}$ in the development of optically pumped organic semiconductor lasers (OSLs), with the ultimate goal of achieving electrically pumped lasing. Efforts to create an electrically pumped OSL are driven by the broad wavelength tunability and high temperature stability of their emission characteristics that have been demonstrated in optically pumped devices. ${ }^{3}$ Several factors have prevented the realization of an electrically pumped OSL, including singlet-triplet and singlet-polaron annihilation, ${ }^{4}$ increased cavity loss resulting from polaron and excited state absorption, ${ }^{5}$ and high waveguide loss due to incorporation of injecting electrodes into the cavity. ${ }^{1}$

In contrast to optical excitation, electrical injection introduces polarons in addition to forming three triplet excitons for every singlet. ${ }^{6,7}$ Both singlet-triplet and singlet-polaron annihilation reactions ${ }^{8,9}$ can dramatically quench the radiative singlet density under high electrical injection. ${ }^{4}$ Quenching by these species is worsened under electrical excitation since triplet and polaron densities are $\sim 1000$ times higher than the singlet density due to their comparatively longer lifetimes $(\geqslant 10 \mu \mathrm{s})$. Attaining high singlet density in the absence of triplets and polarons, as in the case of optically pumped OSLs, is crucial to reaching the lasing threshold. ${ }^{4}$

Here, we investigate the recombination dynamics of metastable geminate polaron-pair (PP) states by monitoring the electric-field-induced delayed fluorescence produced by the guest/host system of Coumarin-6 (C6) doped into $4,4^{\prime}$-bis $(N$-carbazolyl)biphenyl (CBP). We use low intensity, continuous-wave $(\mathrm{cw})$ optical pumping in the presence of a pulsed electric field to accumulate a high density of metastable PP states over time, that are then rapidly reconverted

\footnotetext{
${ }^{a)}$ Electronic mail: stevefor@umich.edu
}

into excitons upon removal of the electric field, leading to peak singlet densities of $\sim 4 \times 10^{15} \mathrm{~cm}^{-3}$. This suggests a method to reach laser threshold excitation densities ${ }^{3}$ $\left(\sim 10^{17} \mathrm{~cm}^{-3}\right)$ by continuously pumping with a low intensity $\left(<1 \mathrm{~W} / \mathrm{cm}^{2}\right)$ optical source. In comparison with direct electrical injection, free polaron densities should be reduced by this process, and triplet exciton formation is expected to be minimal based on previous work concerning the spinconserving nature of geminate pair recombination. ${ }^{8,10}$

Field-induced delayed fluorescence has been observed previously in ladder-type poly(para-phenylene) polymers. ${ }^{10,11}$ In those studies, samples were excited by a laser pulse in the presence of an electric field, and at times long after the natural photoluminescent (PL) decay, a burst of luminescence was observed upon removal of the field. It was concluded that a portion of the initially generated singlet excitons forms interchain PPs that can be stabilized against geminate recombination by the external electric field, which acts to screen the PP Coulomb potential. When the field is removed, rapid reformation into singlet excitons occurs, which then radiatively decay in a fluorescence burst. Here, we study this phenomenon in the small molecule guest/host system consisting of $1 \mathrm{wt} \% \mathrm{C} 6$ doped into the host material CBP.

Devices consisted of a symmetric organic insulator $(50 \mathrm{~nm}) /$ organic semiconductor active layer $(100 \mathrm{~nm}) /$ organic insulator $(50 \mathrm{~nm})$ stack sandwiched between two electrodes to form a capacitor. Commercially available Teflon AF was used for the insulating layers due to its high thin-film dielectric breakdown strength $(>2 \mathrm{MV} / \mathrm{cm}$ ) (Ref. 12) and optical transparency in the ultraviolet. The organic layers were thermally evaporated in sequence onto a precleaned ${ }^{13}$ indium tin oxide (ITO) coated glass substrate under a vacuum of $10^{-7}$ Torr. The active layer 


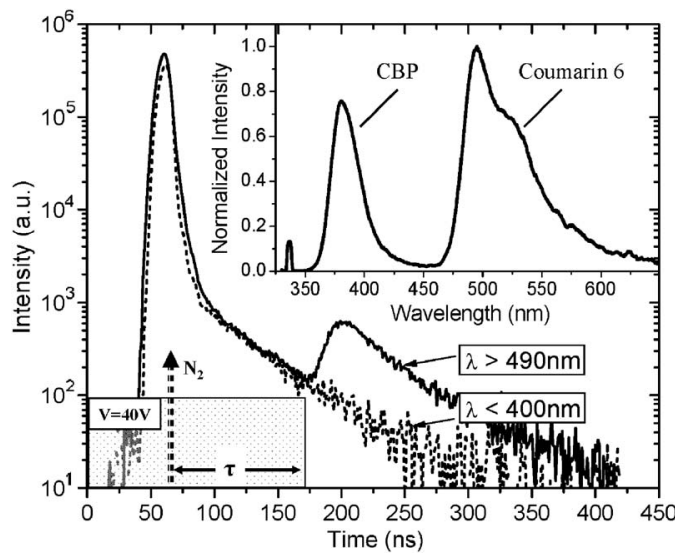

FIG. 1. Luminescence transients for $\operatorname{CBP}(\lambda<400 \mathrm{~nm}$, dashed line $)$ and C6 $(\lambda>490 \mathrm{~nm}$, solid line) measured in the vicinity of voltage turnoff. The timing relationship between excitation and voltage pulse is shown at lower left. Inset: Spectrum of the prompt photoluminescence.

consists of CBP coevaporated with C6 at a 100:1 weight ratio. The devices were completed by depositing a $100 \mathrm{~nm}$ thick $\mathrm{Al}$ cathode through a shadow mask with an array of $1 \mathrm{~mm}$ diameter openings. For testing, samples were loaded into an electrically and optically accessible cryostat evacuated to $50 \mathrm{mTorr}$. Luminescent transients were collected by focusing light from each device into a Hamamatsu C4334 streak camera. Electrical pulses were provided at a rate of $17 \mathrm{~Hz}$ by an HP4114A pulse generator, connected in parallel to the $50 \Omega$ termination of an oscilloscope to monitor the voltage transient. Device capacitances were measured to be $\sim 260 \mathrm{pF}$ at $1 \mathrm{MHz}$, resulting in a $R C$ time constant of $\sim 13$ ns.

Approximately $1 \mu$ s after the application of a $V=40 \mathrm{~V}$ pulse, a $\lambda=337 \mathrm{~nm}$ wavelength $\mathrm{N}_{2}$ laser pulse (600 ps duration, $6.8 \mu \mathrm{J}$ energy, focused to a $1 \mathrm{~mm}$ diameter spot) was incident on the device through the ITO contact. This wavelength is strongly absorbed in CBP $\left(\alpha=2 \times 10^{5} \mathrm{~cm}^{-1}\right.$ at $337 \mathrm{~nm})$, but not in C6 $\left(\lambda_{p}=450 \mathrm{~nm}\right)$. Accounting for the dielectric constants of the organic layers $\left(\varepsilon_{\text {Teflon }}=1.9, \varepsilon_{\text {org }}\right.$ $=3.0$ ), this applied voltage translates into a uniform field of $1.6 \mathrm{MV} / \mathrm{cm}$ across the active layer. The streak camera detection window was synchronized to observe the point of voltage turnoff, occurring at a time $\tau$ following laser excitation.

As shown in Fig. 1 for $\tau=115 \mathrm{~ns}$, a burst of only C6 luminescence appears (rise time $\sim 20 \mathrm{~ns}$ ) upon field removal, independent of bias polarity. The large fraction of CBP luminescence in the prompt spectrum (Fig. 1, inset) arises from incomplete $\mathrm{CBP} \rightarrow \mathrm{C} 6$ Förster transfer that results from donor/acceptor spectral mismatch. Note that the long lifetime component of the CBP transient is likely the result of repopulation of the CBP singlet from triplet-triplet annihilation. ${ }^{14}$ The $\mathrm{C} 6$ transient also contains this component as a result of the $\mathrm{CBP} \rightarrow \mathrm{C} 6$ transfer.

After background subtracting the long-time component from the C6 transient, we find that the burst decay is monoexponential with lifetime $\tau_{F}=13 \pm 2 \mathrm{~ns}$. This is significantly longer than the natural lifetime of $\mathrm{C} 6, \tau_{\mathrm{C} 6}$ $=2.1 \pm 0.1 \mathrm{~ns}$, measured in these devices. However, it corresponds well with the calculated $R C$ time constant. Thus, for short holding times $(\tau<10 \mu \mathrm{s})$, the burst dynamics are likely limited by the discharge of the electric field across the device. $^{11}$

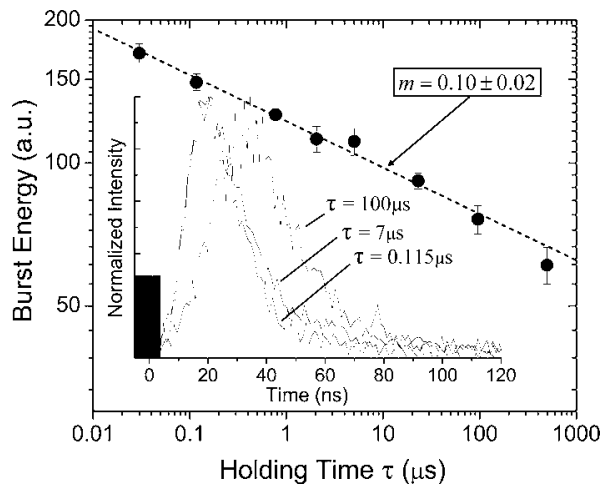

FIG. 2. Decay of the time-integrated burst intensity vs holding electric field time $\tau$. The decay is fitted with a power law $\tau^{-m}$, where $m=0.10 \pm 0.02$. Inset: Fluorescent burst transients for $\tau=0.115,7$, and $100 \mu \mathrm{s}$.

Figure 2 shows the energy of the C6 burst as a function of $\tau$. The burst energy declines over four orders of magnitude in time according to $\tau^{-m}$, where $m \sim 0.1$. This is qualitatively similar to the behavior found in polymers; ${ }^{11,15-17}$ however, the exponent in that case was $m \sim 0.5$. The slow power law decay and the low degree of dispersion in burst transients with variation in $\tau$ are reflective of the stability of the PPs against dissociation. Indeed, energetic disorder is crucial to the burst phenomenon since PPs that relax into the tail of the density of states have their motion restricted, increasing the likelihood that they will remain correlated while in the electric field.

The field-delayed burst was not observed in neat CBP films. Nor does it occur in CBP-C6 devices if the excitation wavelength is below the optical gap of CBP, where only C6 molecules are excited. Thus, the use of a guest/host system is critical to generate the stable PP states that create the fluorescence burst. Here, the energetic difference in the highest occupied molecular orbital (HOMO) levels of C6 and CBP (5.4 and $6.3 \mathrm{eV}$, respectively ${ }^{18,19}$ ) provides a driving force to break the strongly bound $\left[E_{B} \sim 1 \mathrm{eV}\right.$ (Ref. 20)] CBP Frenkel excitons. The hole is then localized on the C6 HOMO in the potential well created by the surrounding CBP molecules. This further stabilizes the PP against complete dissociation, since only the low mobility electron is capable of hopping out of the mutual Coulomb potential.

Due to the sublinear decay in density, PPs can асситиlate at a rate of roughly $\tau^{1-m}$ by continuous optical pumping. To accomplish this, we use the $\lambda=325 \mathrm{~nm}$ line of a cw $\mathrm{HeCd}$ laser, focused to a $1 \mathrm{~mm}$ diameter spot $\left(0.9 \mathrm{~W} / \mathrm{cm}^{2}\right)$ on the same devices as in the $\mathrm{N}_{2}$ pumped experiments. Voltage pulses of $45 \mathrm{~V}$ were superimposed upon a $-5 \mathrm{~V}$ dc bias. The interpulse reverse bias offset removes free charges resulting from fully dissociated PPs generated during the pulses by aiding their recombination. For detection, sample luminescence is filtered (long pass cutoff at $\lambda>450 \mathrm{~nm}$ ) to isolate the C6 emission. The PL is focused onto a Hamamatsu C5460 Si avalanche photodiode (APD)/preamplifier module $\left(f_{c}=10 \mathrm{MHz}\right)$.

Figure 3(a) shows the APD output for $\tau=10,20,40$, and $80 \mu$ s. A postpulse burst of luminescence that grows in proportion to $\tau$ is observed. A magnified view of the increase in burst intensity is shown in the inset; the burst transients are limited by the APD frequency response.

We quantify the intensity of the burst through the enhancement factor $M$, equal to the ratio of the burst intensity, $I_{\text {burst }}$, to the steady-state luminescence intensity, $I_{\mathrm{SS}}$. Figure 

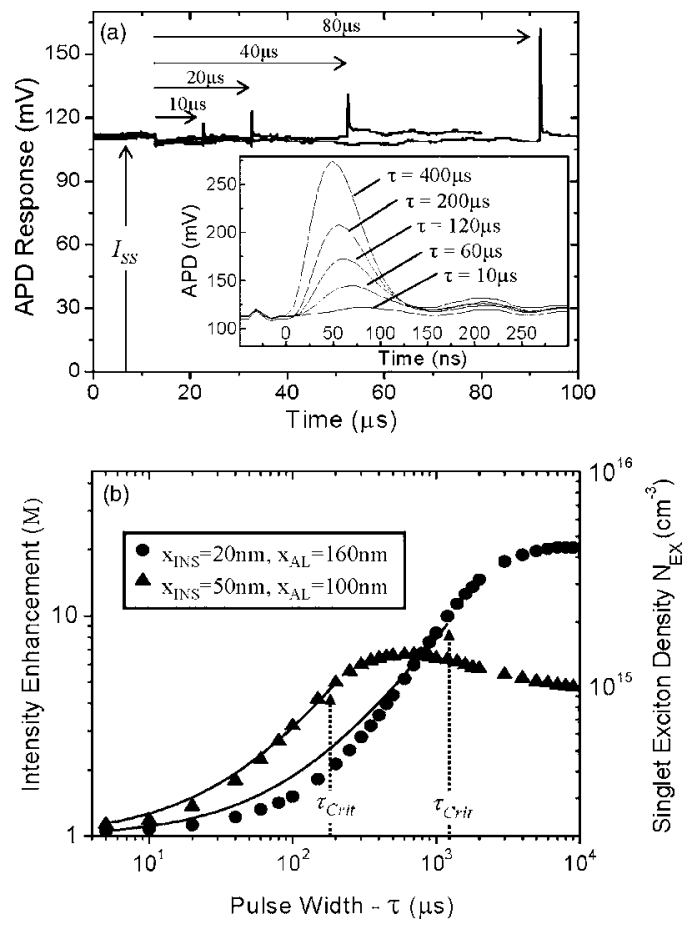

FIG. 3. (a) Measured APD output showing the growth in burst intensity for $\tau=10,20,40$, and $80 \mu$ s. Inset: Burst transients shown at increased time resolution. (b) Plot of $M$, for two devices with different insulating barrier thicknesses $x_{\text {ins. }}$. The solid lines show fits to Eq. (2) with $m=0.1$ and $N_{\mathrm{PP} 0} / N_{\mathrm{SS}}=0.014 \pm 0.002$ and $0.033 \pm 0.003$ for $x_{\mathrm{ins}}=20 \mathrm{~nm}$ and $x_{\mathrm{ins}}=50 \mathrm{~nm}$, respectively.

3(b) shows $M$ plotted versus pulse width $\tau$ for two devices of differing insulating barrier thickness, $x_{\text {ins }}$, but with identical total device thicknesses. Here, the excited state density of the burst, $N_{\text {ex }}$, shown on the right-hand scale, is calculated by estimating the steady-state C6 singlet density $N_{\mathrm{SS}}$ as

$$
N_{\mathrm{SS}}=\frac{I_{0}(\lambda / h c)[0.86] \phi_{\mathrm{C} 6} \tau_{\mathrm{C} 6}}{2 L_{\mathrm{abs}}}
$$

and scaling as $N_{\text {ex }}=M N_{\text {SS. }}$. In Eq. (1), $I_{0}$ is the incident laser intensity $\left(W / \mathrm{cm}^{2}\right)$ and $h$ is Planck's constant. Since the active layer thickness, $x_{\mathrm{al}}=100 \mathrm{~nm}$, is double the CBP absorption length, $L_{\mathrm{abs}}=50 \mathrm{~nm}$ at $\lambda=325 \mathrm{~nm}$, roughly $\left(1-\exp \left(-x_{\mathrm{al}} / L_{\mathrm{abs}}\right)=0.86\right)$ of the incident pump is absorbed in a single pass through the active layer, making cathode reflection and other microcavity effects negligible. The fraction, $\phi_{\mathrm{C} 6}=78 \%$, of $\mathrm{CBP}$ excitations is transferred to $\mathrm{C} 6$ molecules, as estimated from the relative magnitudes of CBP and C6 emission in the PL spectrum (see Fig. 1, inset).

The early $\tau$ portions of each curve are fitted using the PP decay power law with $m=0.1$. That is,

$$
M=\frac{N_{\mathrm{SS}}+N_{\mathrm{PP} 0} \tau^{1-m}}{N_{\mathrm{SS}}}=1+\left(N_{\mathrm{PP} 0} / N_{\mathrm{SS}}\right) \tau^{1-m},
$$

where $N_{\mathrm{PP} 0}$ is the number of initially generated singlets that can undergo the singlet $\rightarrow \mathrm{PP} \rightarrow$ singlet chain of conversions. The roll-off onset in $M$ occurring at $\tau_{\text {crit }}$ [see Fig. 3(b)] results from a buildup of space charge over time due to those PPs that completely dissociate. The resulting space charge creates an opposing internal field that cancels the applied field, reducing $M$. Thinning the insulating barriers to $x_{\text {ins }}=20 \mathrm{~nm}$ [Fig. 3(b)] allows some of the accumulated space charge to tunnel out of the device. The result is an improve- ment of $\tau_{\text {crit }}$ by a factor of 6 , and an increase in the peak enhancement to $M=22$, corresponding to a C6 singlet density of $N_{\mathrm{ex}}=4 \times 10^{15} \mathrm{~cm}^{-3}$. Electrical generation of comparable singlet density would require a current density $J>30 \mathrm{~A} / \mathrm{cm}^{2}$, where it has been shown that both pumpinduced absorption ${ }^{5}$ and annihilation losses are already significant. ${ }^{4}$

In summary, we have investigated the decay dynamics of external-field-stabilized geminate polaron pairs in the C6/ CBP guest/host system by monitoring the field-induced delayed fluorescence bursts following voltage turnoff. The PP density decays sublinearly according to $\tau^{-m}$ (with $m \sim 0.1$ ). We show that geminate PPs can be accumulated under continuous optical pumping, and then rapidly $(<10 \mathrm{~ns})$ reconverted into singlet excitons. The cancellation of the external field by an internal space-charge field arising from fully dissociated PPs currently limits the maximum attainable PP density. A peak singlet exciton density of $N_{\text {ex }}=4 \times 10^{15} \mathrm{~cm}^{-3}$ is obtained under low intensity optical pumping $\left(0.9 \mathrm{~W} / \mathrm{cm}^{2}\right)$, with expectations for future improvement toward the ultimate goal of reaching laser threshold when integrated within an optical microcavity.

The authors thank the Air Force Office of Scientific Research (AFOSR) and Universal Display Corp. for support of this work. In addition, they thank R. J. Holmes and B. P. Rand for helpful discussions.

${ }^{1}$ V. G. Kozlov, G. Parthasarathy, P. E. Burrows, V. B. Khalfin, J. Wang, S. Y. Chou, and S. R. Forrest, IEEE J. Quantum Electron. 36, 18 (2000).

${ }^{2}$ N. Tessler, G. J. Denton, and R. H. Friend, Nature (London) 382, 695 (1996).

${ }^{3}$ V. G. Kozlov, V. Bulovic, P. E. Burrows, M. Baldo, V. B. Khalfin, G. Parthasarathy, S. R. Forrest, Y. You, and M. E. Thompson, J. Appl. Phys. 84, 4096 (1998).

${ }^{4}$ M. A. Baldo, R. J. Holmes, and S. R. Forrest, Phys. Rev. B 66, 035321 (2002).

${ }^{5}$ V. G. Kozlov, P. E. Burrows, G. Parthasarathy, and S. R. Forrest, Appl. Phys. Lett. 74, 1057 (1999).

${ }^{6}$ M. A. Baldo, D. F. O'Brien, M. E. Thompson, and S. R. Forrest, Phys. Rev. B 60, 14422 (1999).

${ }^{7}$ M. Segal, M. A. Baldo, R. J. Holmes, S. R. Forrest, and Z. G. Soos, Phys. Rev. B 68, 075211 (2003).

${ }^{8}$ E. J. W. List, C. H. Kim, A. K. Naik, U. Scherf, G. Leising, W. Graupner, and J. Shinar, Phys. Rev. B 6415, 155204 (2001).

${ }^{9}$ E. J. W. List, U. Scherf, K. Mullen, W. Graupner, C. H. Kim, and J. Shinar, Phys. Rev. B 66, 235203 (2002).

${ }^{10}$ M. Reufer, M. J. Walter, P. G. Lagoudakis, B. Hummel, J. S. Kolb, H. G. Roskos, U. Scherf, and J. M. Lupton, Nat. Mater. 4, 340 (2005).

${ }^{11}$ B. Schweitzer, V. I. Arkhipov, and H. Bassler, Chem. Phys. Lett. 304, 365 (1999).

${ }^{12}$ P. P. Budenstein, P. J. Hayes, J. L. Smith, and W. B. Smith, J. Vac. Sci. Technol. 6, 289 (1969).

${ }^{13}$ P. E. Burrows, Z. Shen, V. Bulovic, D. M. McCarty, S. R. Forrest, J. A. Cronin, and M. E. Thompson, J. Appl. Phys. 79, 7991 (1996).

${ }^{14}$ M. Pope and C. Swenberg, Electronic Processes in Organic Crystals and Polymers (Oxford University Press, New York, NY, 1999), Chap. 1.

${ }^{15}$ A. F. Nogueira, I. Montanari, J. Nelson, J. R. Durrant, C. Winder, and N. S. Sariciftci, J. Phys. Chem. B 107, 1567 (2003).

${ }^{16}$ I. Montanari, A. F. Nogueira, J. Nelson, J. R. Durrant, C. Winder, M. A. Loi, N. S. Sariciftci, and C. Brabec, Appl. Phys. Lett. 81, 3001 (2002).

${ }^{17}$ T. Offermans, S. C. J. Meskers, and R. A. J. Janssen, J. Chem. Phys. 119, 10924 (2003).

${ }^{18}$ H. Suzuki and S. Hoshino, J. Appl. Phys. 79, 858 (1996).

${ }^{19}$ M. A. Baldo, S. Lamansky, P. E. Burrows, M. E. Thompson, and S. R. Forrest, Appl. Phys. Lett. 75, 4 (1999).

${ }^{20}$ I. G. Hill, A. Kahn, Z. G. Soos, and R. A. Pascal, Chem. Phys. Lett. 327, 181 (2000). 